\title{
IDEALS AND GREEN'S RELATIONS IN ORDERED SEMIGROUPS
}

\author{
NIOVI KEHAYOPULU
}

Received 25 November 2005; Revised 3 March 2006; Accepted 12 March 2006

Exactly as in semigroups, Green's relations play an important role in the theory of ordered semigroups - especially for decompositions of such semigroups. In this paper we deal with the $\mathscr{I}$-trivial ordered semigroups which are defined via the Green's relation $\mathscr{I}$, and with the nil and $\Delta$-ordered semigroups. We prove that every nil ordered semigroup is $\mathscr{S}$-trivial which means that there is no ordered semigroup which is 0 -simple and nil at the same time. We show that in nil ordered semigroups which are chains with respect to the divisibility ordering, every complete congruence is a Rees congruence, and that this type of ordered semigroups are $\triangle$-ordered semigroups, that is, ordered semigroups for which the complete congruences form a chain. Moreover, the homomorphic images of $\triangle$-ordered semigroups are $\triangle$-ordered semigroups as well. Finally, we prove that the ideals of a nil ordered semigroup $S$ form a chain under inclusion if and only if $S$ is a chain with respect to the divisibility ordering.

Copyright (c) 2006 Hindawi Publishing Corporation. All rights reserved.

\section{Introduction and prerequisites}

In the theory of ordered semigroups - especially for decompositions of ordered semigroups, an important role is played by the Green's relations. In this paper the concepts of $\mathscr{I}$-trivial, nil ordered semigroups, $\triangle$-ordered semigroups and the concept of complete congruences are introduced. The main results of the paper are the following: if $S$ is an ordered semigroup, $A$ an ideal of $S$, and $B$ an idempotent ideal of $A$, then $B$ is an ideal of $S$. For the corresponding result on semigroups, we refer to [2]. An ideal $A$ of an ordered semigroup $S$ is called idempotent if $A=\left(A^{2}\right]$ [6]. An ordered semigroup $S$ is $\mathscr{I}$-trivial if and only if the divisibility relation on $S$ is an order on $S$. The divisibility relation on $S$ is a chain if and only if $S$ is $\mathscr{I}$-trivial and the principal ideals of $S$ form a chain under inclusion. Moreover the principal ideals of $S$ form a chain with respect to the inclusion relation if and only if the ideals of $S$ do so. For the corresponding result on semigroups, we refer to [12]. The nil ordered semigroups are $\mathscr{S}$-trivial. As a consequence, an ordered semigroup cannot be 0 -simple and nil at the same time.

Hindawi Publishing Corporation

International Journal of Mathematics and Mathematical Sciences

Volume 2006, Article ID 61286, Pages 1-8

DOI 10.1155/IJMMS/2006/61286 
We introduce the concept of a complete congruence on an ordered semigroup $(S, \cdot, \leq)$ and the concept of the Rees congruence modulo $A$, where $A$ is an ideal of $S$. A congruence $\rho$ on $(S, \cdot, \leq)$ is called complete if $a \leq b$ implies $(a, a b) \in \rho$. We prove that if $(S, \cdot, \leq)$ is a nil ordered semigroup which is a chain with respect to the divisibility ordering, then every complete congruence on $S$ is a Rees congruence. Furthermore, the ideals of a nil ordered semigroup $S$ form a chain under inclusion if and only if $S$ is a chain with respect to the divisibility ordering. The concept of a $\triangle$-ordered semigroup is defined as follows: an ordered semigroup $S$ is called a $\triangle$-ordered semigroup if the complete congruences on $S$ form a chain under inclusion. We finally prove that the nil ordered semigroups which are chains with respect to the divisibility ordering are $\triangle$-ordered semigroups and that the homomorphic images of $\triangle$-ordered semigroups are $\triangle$-ordered semigroup as well. For the corresponding results on semigroups-without order-we refer to $[11]$.

If $(S, \cdot, \leq)$ is an ordered semigroup and $H \subseteq S$, we denote by $(H]$ the subset of $S$ defined as follows:

$$
(H]:=\{t \in S \mid t \leq h \text { for some } h \in H\}[4] .
$$

A nonempty subset $A$ of an ordered semigroup $(S, \cdot, \leq)$ is called a left (resp., right) ideal of $S$ if the following hold.

(1) $S A \subseteq A$ (resp., $A S \subseteq A$ ).

(2) If $a \in A$ and $S \ni b \leq a$, then $b \in A$ [4].

Equivalent definition.

(1) $S A \subseteq A$ (resp., $A S \subseteq A$ ).

(2) $(A]=A$.

$A$ is called an ideal of $S$ if it is both a left and a right ideal of $S$.

We denote by $L(A), R(A), I(A)$ the left ideal, right ideal, ideal of $S$, respectively, generated by $A(A \subseteq S)$, that is the least, with respect to the inclusion relation, left ideal, right ideal, or ideal of $S$, respectively, containing $A$. As usual, $L(A)$ (resp., $R(A)$ ) coincides with the intersection of all left (resp., right) ideals of $S$ containing $A, I(A)$ with the intersection of all ideals of $S$ containing $A$. It is easy to check the following:

$$
\begin{gathered}
L(A)=(A \cup S A], \quad R(A)=(A \cup A S], \\
I(A)=(A \cup S A \cup A S \cup S A S] \quad \forall A \subseteq S(\text { cf. also [4]). }
\end{gathered}
$$

For $A=\{a\}$, we write $L(a), R(a), I(a)$ instead of $L(\{a\}),(R\{a\}), I(\{a\})$, respectively, and we call them the principal left ideal, principal right ideal, or principal ideal of $S$, respectively, generated by $a(a \in S)$.

We have

$$
\begin{gathered}
L(a)=\{t \in S \mid t \leq a \text { or } t \leq y a \text { for some } y \in S\}, \\
R(a)=\{t \in S \mid t \leq a \text { or } t \leq a x \text { for some } x \in S\}, \\
I(a)=\{t \in S \mid t \leq a \text { or } t \leq y a \text { for some } y \in S \text { or } t \leq a x \text { for some } x \in S \\
\text { or } t \leq x a y \text { for some } x, y \in S\} .
\end{gathered}
$$


It might be noted that if $S$ is a poe-semigroup, that is, an ordered semigroup ( $p o$-semigroup [1]) having a greatest element " $e$ " with respect to the order of $S$, then, for each $a \in S$, the principal ideals $L(a), R(a), I(a)$ are the following:

$$
\begin{gathered}
L(a)=\{t \in S \mid t \leq a \text { or } t \leq \text { ea }\}, \\
R(a)=\{t \in S \mid t \leq a \text { or } t \leq \text { ae }\}, \\
I(a)=\{t \in S \mid t \leq a \text { or } t \leq \text { ea or } t \leq \text { ae or } t \leq \text { eae }\} .
\end{gathered}
$$

The zero of an ordered semigroup $(S, \cdot, \leq)$ is an element of $S$, usually denoted by 0 , such that $0 \leq x$ and $0 x=x 0=0$ for all $x \in S$ [1]. A nonempty subset $A$ of an ordered semigroup $(S, \cdot, \leq)$ is called a subsemigroup of $S$ if $(A, \cdot)$ is a semigroup, that is, if $A^{2} \subseteq A$.

Remark 1.1. If $(S, \cdot, \leq)$ is an ordered semigroup and $A$ a left (resp., right) ideal of $S$, then $A^{2} \subseteq S A \subseteq A$ (resp., $A^{2} \subseteq A S \subseteq A$ ), so $(A, \cdot)$ is a subsemigroup of $S$. Then $(A, \cdot)$ is a semigroup, and $(A, \cdot, \leq)$ is an ordered semigroup.

Definition 1.2. Let $(S, \cdot, \leq)$ be an ordered semigroup and $A$ an ideal of $S$. $B$ is an ideal of $A$, if $B$ is an ideal of the ordered semigroup $(A, \cdot, \leq)$.

Definition 1.3. An equivalence relation $\rho$ on an ordered semigroup $S$ is called a left (resp., right) congruence on $S$ if $(a, b) \in \rho$ implies $(c a, c b) \in \rho$ (resp., $(a c, b c) \in \rho$ ) for every $c \in S$. An equivalence relation $\rho$ on $S$ is called a congruence on $S$ if it is both a left and a right congruence on $S$.

Definition 1.4. Let $(S, \cdot, \leq)$ be an ordered semigroup. A congruence $\rho$ on $S$ is called complete if $a \leq b$ implies $(a, a b) \in \rho$.

The concept of complete semilattice congruences on an ordered semigroup has been introduced in [7]. A semilattice congruence on $S$ is a congruence $\rho$ on $S$ such that $\left(a^{2}, a\right) \in$ $\rho$ and $(a b, b a) \in \rho$ for each $a, b \in S$.

Definition 1.5. In an ordered semigroup $S$ the Green's relations $\mathscr{L}, \mathscr{R}, \mathscr{I}, \mathscr{H}, \mathscr{D}$ are defined as follows: $a \mathscr{L} b$ if $L(a)=L(b), a \mathscr{R} b$ if $R(a)=R(b), a \mathscr{I} b$ if $I(a)=I(b), \mathscr{H}=\mathscr{L} \cap \mathscr{R}$ and $\mathscr{D}=\mathscr{L} \vee \mathscr{R}$, that is, the smallest equivalence relation of $S$ containing both $\mathscr{L}$ and $\mathscr{R}$.

It is easy to check that $\mathscr{L}$ is a right congruence and $\mathscr{R}$ is a left congruence on $S$. This has been proved in case of poe-semigroups in [3].

A mapping $f$ of an ordered semigroup $(S, \cdot, \leq)$ into an ordered semigroup $(T, *, \preceq)$ is called a homomorphism if $f(a b)=f(a) * f(b)$ and $a \leq b$ implies $f(a) \preceq f(b)$ for each $a, b \in S[8]$.

\section{The main results}

Lemma 2.1. If $(S, \cdot, \leq)$ is an ordered semigroup, $A$ an ideal of $S$, and $B$ an ideal of $A$, then $(B]=B$.

Proof. We have

$$
(B]:=\{t \in S \mid t \leq b \text { for some } b \in B\} .
$$


If $t \in B$, then, since " $\leq$ " is reflexive on $S$, we have $t \in(B]$. If $t \in(B]$, then $t \leq b$ for some $b \in B \subseteq A$. Since $S \ni t \leq b \in A$ and $A$ is an ideal of $S$, we have $t \in A$. Since $A \ni t \leq b \in B$ and $B$ is an ideal of $A$, we have $t \in B$. Then $(B] \subseteq B$, and thus $(B]=B$.

Lemma 2.2 [4]. Let $S$ be an ordered semigroup. Then the following hold.

(1) $A \subseteq(A]$ for all $A \subseteq S$.

(2) If $A \subseteq B \subseteq S$, then $(A] \subseteq(B]$.

(3) $(A](B] \subseteq(A B]$ for all $A, B \subseteq S$.

(4) $((A]]=(A]$ for all $A \subseteq S$.

(5) If $A, B$ are ideals of $S$, then $(A B], A \cup B$ are ideals of $S$.

(6) In particular, if $S$ is an ordered semigroup, then the set (SaS] is an ideal of $S$ for all $a \in S$.

Proposition 2.3. Let $(S, \cdot, \leq)$ be an ordered semigroup, $A$ an ideal of $S$, and $B$ an ideal of $A$ such that $B=\left(B^{2}\right]$. Then $B$ is an ideal of $S$.

Proof. First of all, by Lemma 2.1, we have $(B]=B$. Moreover,

$$
\begin{aligned}
B S & =\left(B^{2}\right] S=\left(B^{2}\right](S] \subseteq\left(B^{2} S\right]=(B(B S)] \\
& \subseteq(B(A S)] \subseteq(B A] \subseteq(B]=B(\text { cf. also Lemma 2.2 }) .
\end{aligned}
$$

In a similar way we get $S B \subseteq B$. Since $B S \subseteq B, S B \subseteq B$ and $(B]=B, B$ is an ideal of $S$.

Definition 2.4. An ordered semigroup $S$ is called $\mathscr{I}$-trivial if the Green's equivalence relation $\mathscr{I}$ on $S$ is the equality relation on $S$, that is, if $I(a)=I(b)$ implies $a=b$.

Definition 2.5. Let $S$ be an ordered semigroup and $a, b \in S$. Say that $b$ divides $a$ (or $a$ is divisable by $b$ ), and write $b \mid a$, if $a \in I(b)$.

Definition 2.6. Let $S$ be an ordered semigroup. A relation “ $\preccurlyeq$ ” is defined on $S$ as follows:

$$
\begin{aligned}
\preccurlyeq & :=\{(a, b) \in S \times S|b| a\} \\
& =\{(a, b) \in S \times S \mid a \in I(b)\} .
\end{aligned}
$$

The relation “ $\preccurlyeq$ ” is called the divisibility relation on $S$.

Proposition 2.7. Let $(S, \cdot, \leq)$ be an ordered semigroup. If $S$ is $\mathscr{I}$-trivial, then the divisibility relation on $S$ is an order on $S$. Conversely, if the divisibility relation “ $\preccurlyeq$ " on $S$ is symmetric, then $S$ is $\mathscr{I}$-trivial.

Proof. ( $\Rightarrow$ ) If $a \in S$, then $a \in I(a)$, so $(a, a) \in \preccurlyeq$. Let $(a, b) \in \preccurlyeq$ and $(b, a) \in \preccurlyeq$. Since $a \in$ $I(b)$ and $b \in I(a)$, we have $I(a) \subseteq I(b)$ and $I(b) \subseteq I(a)$, that is, $I(a)=I(b)$. Since $S$ is $\Phi_{\text {- }}$ trivial, we have $a=b$. If $(a, b) \in \preccurlyeq,(b, c) \in \preccurlyeq$, then $a \in I(b), b \in I(c)$, so $a \in I(b) \subseteq I(c)$, $a \in I(c)$, and $(a, c) \in \preccurlyeq$.

$(\Leftarrow)$ Let the divisibility relation " $\preccurlyeq$ " on $S$ be symmetric and let $a, b \in S$ such that $I(a)=$ $I(b)$. Since $a \in I(b)$ and $b \in I(a)$, we have $(a, b) \in \preccurlyeq$ and $(b, a) \in \preccurlyeq$, so $a=b$.

Corollary 2.8. An ordered semigroup $S$ is $\mathscr{S}$-trivial if and only if the divisibility relation on $S$ is an order on $S$. 
PROPOSITION 2.9. Let $S$ be an ordered semigroup. The following statements are equivalent.

(i) $S$ is $\mathscr{I}$-trivial and the principal ideals of $S$ form a chain under inclusion.

(ii) The divisibility relation "ß” on $S$ is a chain.

Proof. (i) $\Rightarrow$ (ii). By Proposition 2.7 , the divisibility relation “ $\preccurlyeq$ ” is an order on $S$. Let now $a, b \in S$. By (i), we have $I(a) \subseteq I(b)$ or $I(b) \subseteq I(a)$. If $I(a) \subseteq I(b)$, then $a \in I(b)$, and $(a, b) \in \preccurlyeq$. If $I(b) \subseteq I(a)$, then $b \in I(a)$, and $(b, a) \in \preccurlyeq$. So " $\preccurlyeq$ " is a chain.

(ii) $\Rightarrow$ (i). Since “ $\preccurlyeq$ " is an order on $S$, by Proposition $2.7, S$ is $\mathscr{I}$-trivial. Let now $a, b \in S$. Since "§" is a chain, we have $(a, b) \in \preccurlyeq$ or $(b, a) \in \preccurlyeq$. If $(a, b) \in \preccurlyeq$, then $a \in I(b)$, so $I(a) \subseteq$ $I(b)$. If $(b, a) \in \preccurlyeq$, then $b \in I(a)$, so $I(b) \subseteq I(a)$. Hence the principal ideals of $S$ form a chain.

Proposition 2.10. Let $S$ be an ordered semigroup. The principal ideals of $S$ form a chain with respect to the inclusion relation if and only if the ideals of $S$ do so.

Proof. $(\Rightarrow)$ Let $A, B$ be ideals of $S$ such that $A \nsubseteq B$. Then $B \subseteq A$. Indeed, let $b \in B$. We consider an element $a \in A$ such that $a \notin B$. By hypothesis, we have $I(a) \subseteq I(b)$ or $I(b) \subseteq$ $I(a)$. If $I(a) \subseteq I(b)$, then $a \in I(b) \subseteq B$, so $a \in B$ which is impossible. Thus we have $I(b) \subseteq$ $I(a)$, from which $b \in I(a) \subseteq A$.

The implication " $\Leftarrow$ " is obvious.

Definition 2.11 (cf. also [5, 10]). An element $a$ of an ordered semigroup $S$ with zero is called nilpotent if there exists an element $n \in \mathbb{N}$ such that $a^{n}=0$. An ordered semigroup $S$ having a zero element 0 is called nil if every element of $S$ is nilpotent, that is, if for each $a \in S$, there exists $n \in \mathbb{N}$ such that $a^{n}=0 . S$ is called nilpotent if there exists $n \in \mathbb{N}$ such that $S^{n}=\{0\} . \mathbb{N}=\{1,2, \ldots\}$ is the set of natural numbers.

If an ordered semigroup $S$ is nilpotent, then it is clearly nil.

Notation 2.12. For convenience, we use the notation $S^{1}:=S \cup\{1\}$, where 1 is a symbol, such that $1 a:=a, a 1:=a$ for each $a \in S$ and $11:=1$.

Theorem 2.13. Every nil ordered semigroup is $\mathscr{I}$-trivial.

Proof. Let $a, b$ be two elements of an ordered nil semigroup $(S, \cdot, \leq)$ such that $I(a)=I(b)$. Since $a \in I(b)$, we have $a \leq x b y$ for some $x, y \in S^{1}$. Since $b \in I(a)$, we have $b \leq z a t$ for some $z, t \in S^{1}$. Then

$$
\begin{aligned}
a \leq x(z a t) y & =(x z) a(t y) \leq(x z) x z a t y(t y)=(x z)^{2} a(t y)^{2} \\
& \leq(x z)^{2} x z a t y(t y)^{2}=(x z)^{3} a(t y)^{3} \leq(x z)^{3} x z a t y(t y)^{3}=(x z)^{4} a(t y)^{4} .
\end{aligned}
$$

Continuing this way, we have $a \leq(x z)^{n} a(t y)^{n}$ for each $n \in \mathbb{N}$. Since $S$ is nil, there exists $n \in \mathbb{N}$ such that $(x z)^{n}=0$. Then $a=0$, and $b=0$, so $a=b$. Thus $S$ is $\mathscr{I}$-trivial.

It might be noted that using the notation $S^{1}$, we avoid to check each of the 16 (different) cases arising from $b \in I(a)$ and $a \in I(b)$, separately.

Definition 2.14 [9]. An ordered semigroup $S$ having a zero element 0 is called 0 -simple if $S^{2} \neq\{0\}$ and $S$ and $\{0\}$ are the only ideals of $S$.

Corollary 2.15. An ordered semigroup S cannot be 0-simple and nil (at the same time). 
Proof. Suppose it is. If $S=\{0\}$, then $S^{2}=\{0\}$ which is impossible. So there is at least one nonzero element $a$ in $S$. Suppose $b$ is another nonzero element of $S$. We have $I(a) \neq\{0\}$ and $I(b) \neq\{0\}$. Since $S$ is 0 -simple, the ideals $S$ and $\{0\}$ are the only ideals of $S$. So we have $I(a)=S$ and $I(b)=S$. Since $S$ is nil, by Theorem 2.13, it is $\mathscr{I}$-trivial. Then, since $I(a)=I(b)$, we have $a=b$. Thus we have $S=\{0, a\}$ for some $a \in S, a \neq 0$. Then we have $a^{2}=0$. Indeed, if $a^{2}=a$, then $a^{n}=a$ for each $n \in \mathbb{N}$. Since $S$ is nil, there exists $n \in \mathbb{N}$ such that $a^{n}=0$. Then we have $a=0$ which is impossible. Since $S^{2}=\left\{0, a^{2}\right\}$ and $a^{2}=0$, we have $S^{2}=\{0\}$ which is impossible.

Notation 2.16. If $S$ is an ordered semigroup and $A$ an ideal of $S$, we denote by $\rho_{A}$ the relation on $S$ defined as follows:

$$
\rho_{A}:=\{(x, y) \in S \times S \mid x=y \text { or } x, y \in A\} .
$$

It is easy to check that the relation $\rho_{A}$ is a congruence on $S$.

Definition 2.17. For an ordered semigroup $S$ and an ideal $A$ of $S$, the congruence $\rho_{A}$ is called the Rees congruence on $S$ modulo $A$. $S$ is called the ideal extension of $A$ by $S / \rho_{A}$.

THeOREM 2.18. Let $S$ be a nil ordered semigroup which is a chain with respect to the divisibility ordering. Then every complete congruence on $S$ is a Rees congruence.

Proof. Let $\rho$ be a complete congruence on $S$. We define

$$
A:=\{a \in S \mid(a, 0) \in \rho\}
$$

The set $A$ is an ideal of $S$. Indeed, let $a \in A, b \in S$. Since $a \in A$, we have $(a, 0) \in \rho$. Then $(a b, 0 b) \in \rho,(a b, 0) \in \rho$, so $a b \in A$. Let $a \in S, b \in A$. Since $(b, 0) \in \rho$, we have $(a b, 0) \in \rho$, so $a b \in A$. Let $a \in A$ and $S \ni b \leq a$. Then $b \in A$. Indeed, since $\rho$ is complete and $b \leq a$, we have $(b, b a) \in \rho$. Since $a \in A$, we have $(a, 0) \in \rho$, then $(b a, 0) \in \rho$. Since $(b, b a) \in \rho$ and $(b a, 0) \in \rho$, we have $(b, 0) \in \rho$, thus we have $b \in A$.

For the ideal $A$ of $S$, we consider the Rees congruence

$$
\rho_{A}:=\{(x, y) \in S \times S \mid x=y \text { or } x, y \in A\}
$$

defined above. We have $\rho=\rho_{A}$. Indeed, let $(a, b) \in \rho$. Since $a, b \in S$ and $S$ is a chain with respect to the divisibility ordering, we have $(a, b) \in \preccurlyeq$ or $(b, a) \in \preccurlyeq$, namely, $a \in I(b)$ or $b \in I(a)$. Suppose $a \in I(b)$. Then $a \leq x b y$ for some $x, y \in S^{1}$. Then, since $\rho$ is a complete congruence on $S$, we have $(a, a x b y) \in \rho$. Then $(a(x b y), a x b y(x b y)) \in \rho$, so $\left(a, a(x b y)^{2}\right) \in$ $\rho$. Then, since $\left(a(x b y), a(x b y)^{3}\right) \in \rho$, we have $\left(a, a(x b y)^{3}\right) \in \rho$. Continuing this way, we have $\left(a, a(x b y)^{n}\right) \in \rho$ for every $n \in \mathbb{N}$. Since $S$ is nil, there exists $n \in \mathbb{N}$ such that $(x b y)^{n}=$ 0 . Then we have $(a, 0) \in \rho$, and $a \in A$. Since $(b, a) \in \rho$ and $(a, 0) \in \rho$, we have $(b, 0) \in \rho$, and $b \in A$. Since $a, b \in A$, we have $(a, b) \in \rho_{A}$. If $b \in I(a)$, similarly, we get $a, b \in A$, so $(a, b) \in \rho_{A}$. Thus we have $\rho \subseteq \rho_{A}$.

Conversely, let $(a, b) \in \rho_{A}$. Then $a=b$ or $a, b \in A$. If $a=b$, then $(a, b)=(a, a) \in \rho$. If $a, b \in A$, then $(a, 0) \in \rho$ and $(b, 0) \in \rho$, from which $(a, b) \in \rho$. Thus $\rho_{A} \subseteq \rho$.

Hence, for the ideal $A$ of $S$, we have $\rho=\rho_{A}$, and the proof is complete. 
Definition 2.19. An ordered semigroup $S$ is called a $\triangle$-ordered semigroup if the complete congruences on $S$, with respect to the inclusion relation, form a chain.

Proposition 2.20. Let $S$ be a nil ordered semigroup. The ideals of $S$ form a chain under inclusion if and only if $S$ is a chain with respect to the divisibility ordering.

Proof. $(\Rightarrow)$ By Proposition 2.10, the principal ideals of $S$ form a chain under inclusion. Since $S$ is nil, by Theorem 2.13, $S$ is $\mathscr{I}$-trivial. Then, by Proposition $2.9, S$ is a chain with respect to the divisibility ordering.

$(\Leftarrow)$ Let $A, B$ be ideals of $S$ such that $A \nsubseteq B$. Then $B \subseteq A$. Indeed, let $b \in B$, and $a \in A$, $a \notin B$. Since $a, b \in S$, by hypothesis, we have $(a, b) \in \preccurlyeq$ or $(b, a) \in \preccurlyeq$, that is, $a \in I(b)$ or $b \in I(a)$. Since $I(b) \subseteq B$ and $a \notin B$, we have $a \notin I(b)$. Thus we have $b \in I(a) \subseteq A$, and $b \in A$.

Proposition 2.21. Let $S$ be a nil ordered semigroup which is a chain with respect to the divisibility ordering. Then $S$ is a $\triangle$-ordered semigroup.

Proof. Let $\rho, \mu$ be two complete congruences on $S$. Since $S$ is a nil ordered semigroup and it is a chain under the divisibility ordering, by Theorem 2.18, $\rho$ and $\mu$ are Rees congruences on $S$. That is, there exist ideals $A, B$ of $S$ such that $\rho=\rho_{A}$ and $\mu=\rho_{B}$, where

$$
\begin{aligned}
& \rho_{A}:=\{(x, y) \in S \times S \mid x=y \text { or } x, y \in A\}, \\
& \rho_{B}:=\{(x, y) \in S \times S \mid x=y \text { or } x, y \in B\} .
\end{aligned}
$$

By Proposition 2.20, the ideals of $S$ form a chain under inclusion, so we have $A \subseteq B$ or $B \subseteq A$. If $A \subseteq B$, then $\rho_{A} \subseteq \rho_{B}$. Indeed, if $(x, y) \in \rho_{A}$, then $x=y$ or $x, y \in A$, then $x=y$ or $x, y \in B$, which shows that $(x, y) \in \rho_{B}$. By symmetry, if $B \subseteq A$, then $\rho_{B} \subseteq \rho_{A}$. So we have $\rho \subseteq \mu$ or $\mu \subseteq \rho$ and the proof is complete.

Theorem 2.22. Let $(S, \cdot, \leq)$ be a $\triangle$-ordered semigroup, $(T, \cdot, \preceq)$ an ordered semigroup, and $f: S \rightarrow T$ a homomorphism and onto mapping. Then $T$ is a $\triangle$-ordered semigroup.

Proof. Let $\rho, \mu$ be two complete congruences on $T$. Let

$$
\begin{aligned}
& \rho^{*}:=\{(a, b) \in S \times S \mid(f(a), f(b)) \in \rho\}, \\
& \mu^{*}:=\{(a, b) \in S \times S \mid(f(a), f(b)) \in \mu\} .
\end{aligned}
$$

The relations $\rho^{*}$ and $\mu^{*}$ are complete congruences on $S$.

In fact, it is easy to see that the relations $\rho^{*}$ and $\mu^{*}$ are equivalence relations on $S$.

Let now $(a, b) \in \rho^{*}$ and $c \in S$. Then $(a c, b c) \in \rho^{*}$. Indeed, since $(a, b) \in \rho^{*}$, we have $a, b \in S$ and $(f(a), f(b)) \in \rho$. Since $\rho$ is a right congruence on $T$, we have $(f(a) f(c)$, $f(b) f(c)) \in \rho$. Then, since $f$ is a homomorphism, we have $(f(a c), f(b c)) \in \rho$. Since $a c, b c \in S$ and $(f(a c), f(b c)) \in \rho$, we have $(a c, b c) \in \rho^{*}$. Similarly, $\rho^{*}$ is a left congruence on $S$.

$\rho^{*}$ is a complete congruence on $S$. Indeed, let $a, b \in S, a \leq b$. Since $a, b \in S$ and $f$ is a homomorphism, we have $f(a) \preceq f(b)$. Then, since $\rho$ is a complete congruence on $T$, we have $(f(a), f(a) f(b)) \in \rho$. Since $f$ is a homomorphism, we have $(f(a), f(a b)) \in \rho$. Since $a, a b \in S$ and $(f(a), f(a b)) \in \rho$, we have $(a, a b) \in \rho^{*}$. 
Since $S$ is a $\triangle$-ordered semigroup, we have $\rho^{*} \subseteq \mu^{*}$ or $\mu^{*} \subseteq \rho^{*}$. If $\rho^{*} \subseteq \mu^{*}$, then $\rho \subseteq \mu$. Indeed, let $(x, y) \in \rho \subseteq T \times T$. Since $x, y \in T$ and $f$ is onto, there exist $a, b \in S$ such that $f(a)=x, f(b)=y$. Since $a, b \in S$ and $(f(a), f(b)) \in \rho$, we have $(a, b) \in \rho^{*} \subseteq \mu^{*}$. Then $(f(a), f(b)) \in \mu$, so $(x, y) \in \mu$. If $\mu^{*} \subseteq r^{*}$, by symmetry, we have $\mu \subseteq \rho$. So we have $\rho \subseteq \mu$ or $\mu \subseteq \rho$, and $T$ is a $\triangle$-ordered semigroup.

\section{Acknowledgments}

I would like to express my warmest thanks to the Managing Editor of the journal Professor Lokenath Debnath for his interest in my work and for editing and communicating the paper, and to the three referees for their time to read the manuscript very carefully and their useful remarks. This research has been supported by the Special Research Account of the University of Athens (Grant no. 70/4/5630).

\section{References}

[1] G. Birkhoff, Lattice Theory, 3rd ed., American Mathematical Society Colloquium Publications, vol. 25, American Mathematical Society, Rhode Island, 1967.

[2] A. H. Clifford and G. B. Preston, The Algebraic Theory of Semigroups. Vol. I, Mathematical Surveys, no. 7, American Mathematical Society, Rhode Island, 1964.

[3] N. Kehayopulu, On left regular ordered semigroups, Mathematica Japonica 35 (1990), no. 6, 1057-1060.

[4] __ On weakly prime ideals of ordered semigroups, Mathematica Japonica 35 (1990), no. 6, 1051-1056.

[5] _ On ordered semigroups without nilpotent ideal elements, Mathematica Japonica 36 (1991), no. 2, 323-326.

[6] __ On regular ordered semigroups, Mathematica Japonica 45 (1997), no. 3, 549-553.

[7] N. Kehayopulu and M. Tsingelis, $\mathcal{N}$-subsets of ordered semigroups, Partitions and Holomorphic Mappings of Semigroups (E. S. Lyapin, ed.), "Obrazovanie”, St. Petersburg, 1992, pp. 56-63.

[8] __ On subdirectly irreducible ordered semigroups, Semigroup Forum 50 (1995), no. 2, 161177.

[9] _ Characterizations of 0-simple ordered semigroups, Scientiae Mathematicae 3 (2000), no. 3, 339-344.

[10] N. H. McCoy, The Theory of Rings, 5th ed., The Macmillan; New York, Collier-Macmillan, London, 1968.

[11] A. Nagy, Special Classes of Semigroups, Advances in Mathematics (Dordrecht), vol. 1, Kluwer Academic, Dordrecht, 2001.

[12] G. Szász, Eine Charakteristik der Primidealhalbgruppen, Publicationes Mathematicae Debrecen 17 (1970), no. 1-4, 209-213 (1971).

Niovi Kehayopulu: Department of Mathematics, University of Athens,

15784 Panepistimiopolis, Greece

E-mail address: nkehayop@math.uoa.gr 


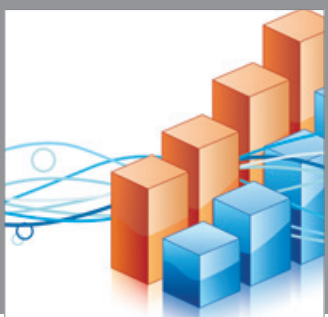

Advances in

Operations Research

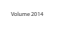

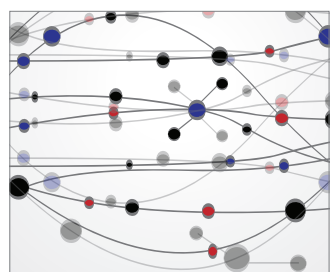

\section{The Scientific} World Journal
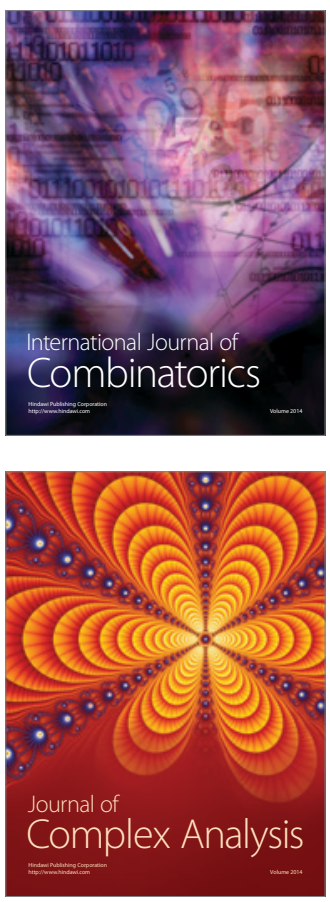

International Journal of

Mathematics and

Mathematical

Sciences
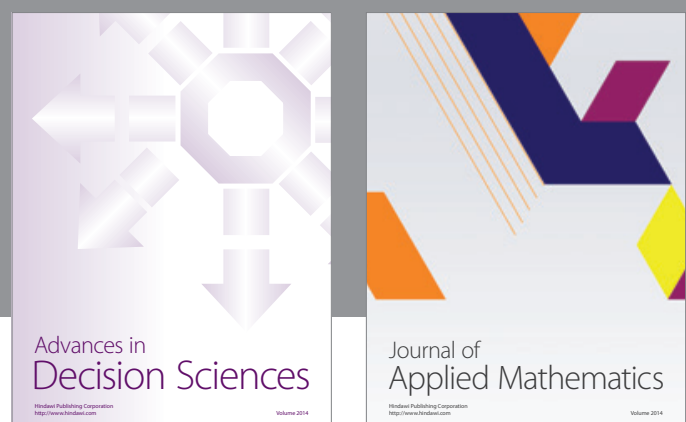

Journal of

Applied Mathematics
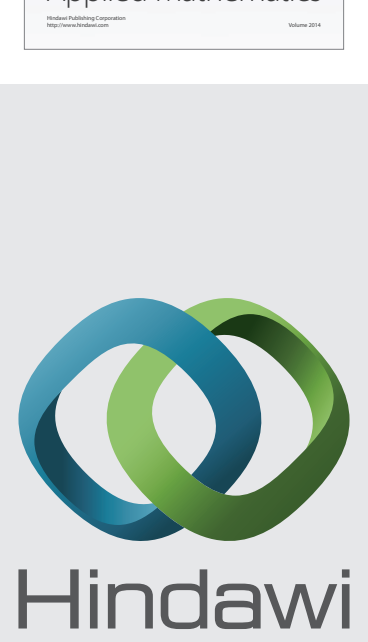

Submit your manuscripts at http://www.hindawi.com
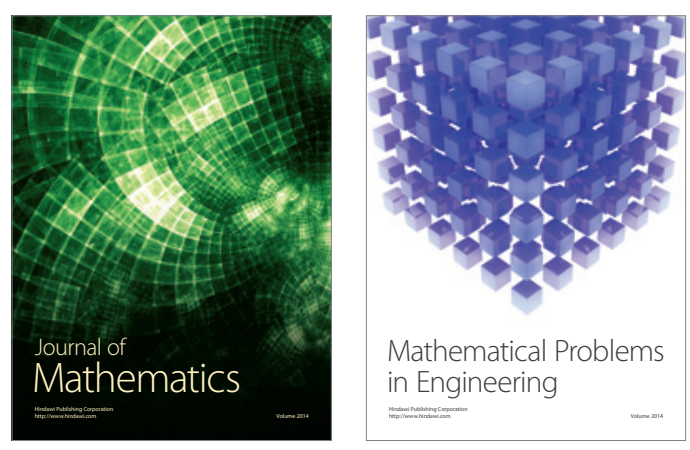

Mathematical Problems in Engineering
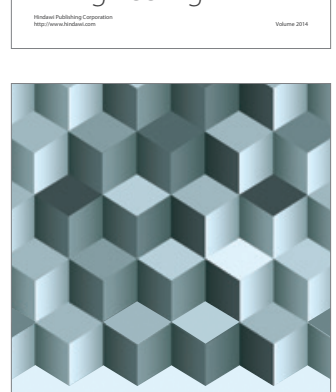

Journal of

Function Spaces
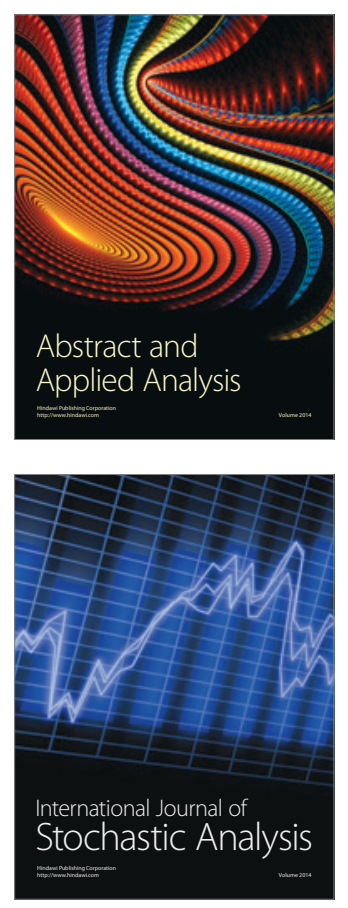

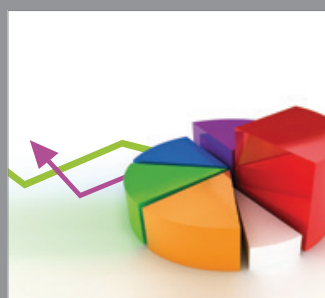

ournal of

Probability and Statistics

Promensencen
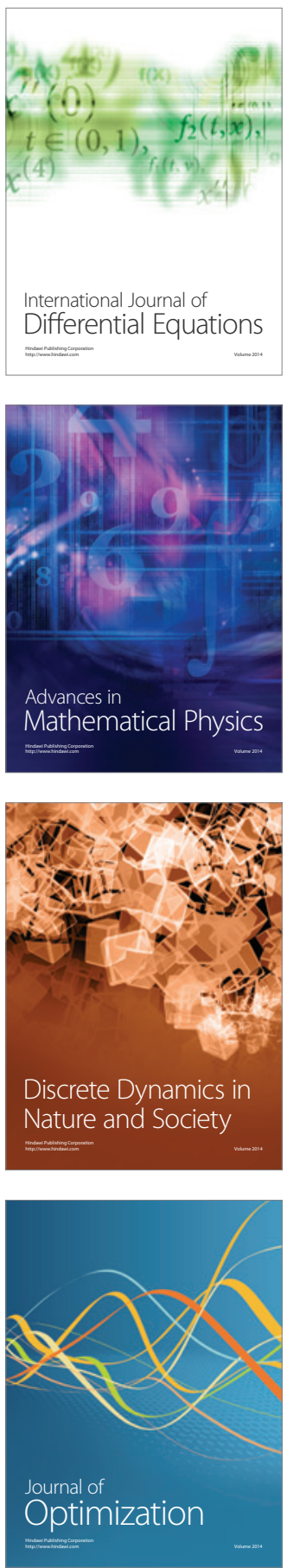